\title{
NSAIDs prevent, but do not reverse, neuronal cell cycle reentry in a mouse model of Alzheimer disease
}

\author{
Nicholas H. Varvel, ${ }^{1,2}$ Kiran Bhaskar, ${ }^{1}$ Maria Z. Kounnas, ${ }^{3}$ Steven L. Wagner, ${ }^{3}$ \\ Yan Yang, ${ }^{2}$ Bruce T. Lamb, ${ }^{1,2,4}$ and Karl Herrup 5 \\ 'Department of Neurosciences, Lerner Research Institute, Cleveland Clinic, Cleveland, Ohio, USA. 'Department of Neurosciences, \\ Case Western Reserve University School of Medicine, Cleveland, Ohio, USA. ${ }^{3}$ Torrey Pines Therapeutics, Inc., La Jolla, California, USA. \\ ${ }^{4}$ Department of Genetics, Case Western Reserve University School of Medicine, Cleveland, Ohio, USA. \\ ${ }^{5}$ Department of Cell Biology and Neuroscience Nelson Biological Laboratories, Rutgers, \\ The State University of New Jersey, Piscataway, New Jersey, USA.
}

\begin{abstract}
Ectopic cell cycle events (CCEs) mark vulnerable neuronal populations in human Alzheimer disease (AD) and are observed early in disease progression. In transgenic mouse models of $\mathrm{AD}, \mathrm{CCEs}$ are found before the onset of $\beta$-amyloid peptide (A $\beta$ ) deposition to form senile plaques, a hallmark of AD. Here, we have demonstrated that alterations in brain microglia occur coincidently with the appearance of CCEs in the R1.40 transgenic mouse model of AD. Furthermore, promotion of inflammation with LPS at young ages in R1.40 mice induced the early appearance of neuronal CCEs, whereas treatment with 2 different nonsteroidal antiinflammatory drugs (NSAIDs) blocked neuronal CCEs and alterations in brain microglia without altering amyloid precursor protein (APP) processing and steady-state A $\beta$ levels. In addition, NSAID treatment of older R1.40 animals prevented new neuronal CCEs, although it failed to reverse existing ones. Retrospective human epidemiological studies have identified long-term use of NSAIDs as protective against AD. Prospective clinical trials, however, have failed to demonstrate a similar benefit. Our use of CCEs as an outcome measure offers fresh insight into this discrepancy and provides important information for future clinical trials, as it suggests that NSAID use in human AD may need to be initiated as early as possible to prevent disease progression.
\end{abstract}

\section{Introduction}

Alzheimer disease (AD), the most common dementing disorder of late life, is now the sixth leading cause of death in the United States (1). The course of the disease is lengthy, and there is currently no effective treatment. A definitive diagnosis of $\mathrm{AD}$ requires postmortem examination of brain tissue for the presence of distinctive $\mathrm{AD}$ histopathology, including neurofibrillary tangles and extracellular deposits of the $\beta$-amyloid peptide (A $\beta$ ) in senile plaques. Activation of microglia and astrocytes, induction of neuronal cell cycle events (CCEs), and regional cell loss are also observed. Understanding the relationship between the various neuropathological hallmarks of $\mathrm{AD}$ in the human brain has proven difficult because of a lack of accurate diagnostic markers; the length of disease progression; and the considerable variation in the duration, severity, symptoms, age of onset, and clinical/pathological correlations.

$\mathrm{AD}$ is one of many neurodegenerative conditions characterized by chronic neuroinflammatory processes. Microglia, the resident immune cells of the brain, are found in a highly activated state in close anatomical proximity to senile plaques within the AD brain, where they secrete numerous proinflammatory cytokines and chemokines $(2,3)$. Recent studies using in vivo imaging have demonstrated that microglia rapidly migrate to newly formed $\mathrm{A} \beta$ deposits in mouse models of $\mathrm{AD}$ and are capable of removing $\mathrm{A} \beta$ fibrils $(4,5)$. However, it remains to be determined whether neuroinflammatory alterations also contribute to early steps in $\mathrm{AD}$ progression. Retrospective epidemiological studies indicate that

Conflict of interest: The authors have declared that no conflict of interest exists. Citation for this article: J. Clin. Invest. 119:3692-3702 (2009). doi:10.1172/JCI39716 chronic, long-term treatment with nonsteroidal antiinflammatory drugs (NSAIDs) decreases the risk for developing AD, which suggests that neuroinflammation may play a pivotal role in early disease processes (6-8). However, thus far, prospective clinical trials with multiple different NSAIDs have failed to demonstrate significant beneficial effects in individuals with existing cognitive impairments characteristic of AD (9). At present, the biological mechanisms underlying the divergent results obtained in the retrospective and prospective NSAID studies remain unclear.

NSAIDs may act via several pathways to influence AD pathogenesis. First, NSAIDs can reduce neuroinflammation via canonical antiinflammatory pathways within the brain. Indeed, chronic administration of NSAIDs reduces neuroinflammation, AD-like brain pathology, and behavioral impairments in transgenic mouse models of AD (10-13). Second, NSAIDs can act as $\gamma$-secretase modulators (GSMs). Acute administration of selective NSAIDs results in production of shorter, less amyloidogenic $A \beta$ peptides both in vitro and in vivo, likely through interactions with the amyloid precursor protein (APP) that influence $\gamma$-secretase cleavage (14-16). Third, NSAIDs may also regulate the levels of $\beta$-secretase through a PPAR $\gamma$-mediated pathway (17). Finally, NSAIDs may act to inhibit the formation of $A \beta$ oligomers and deposits through direct interaction with the $\mathrm{A} \beta$ peptide (16).

Increasing evidence suggests that ectopic expression of cell cycle proteins and DNA synthesis identifies neuronal populations subject to degeneration in $\mathrm{AD}(18-21)$. CCEs are also observed in mild cognitive impairment, the clinical predecessor to AD (22). Together, these results suggest the CCEs identify an early pathogenic process in AD. Highlighting the significance of the 

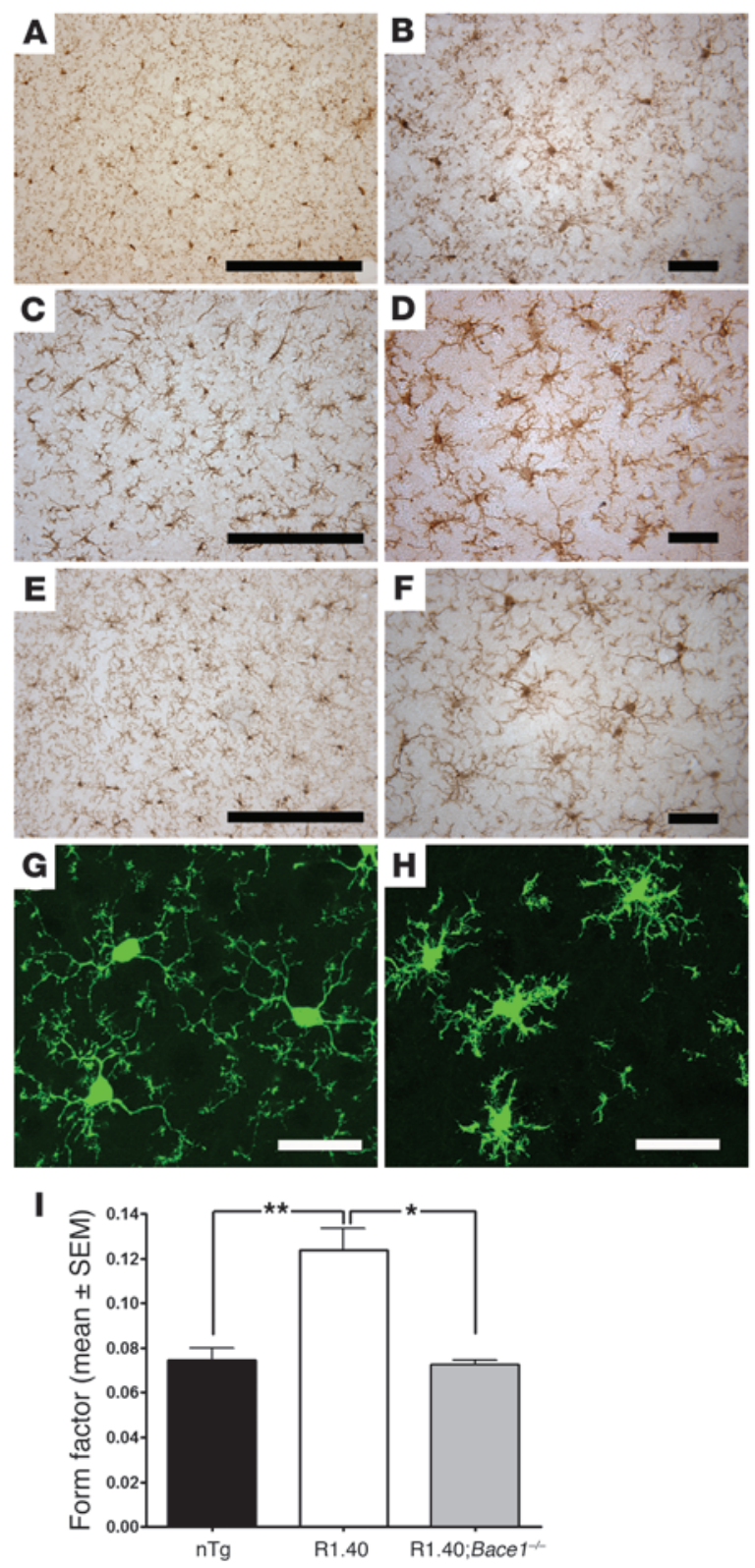

CCEs in the neurobiology of $\mathrm{AD}$, most mouse models that have been examined exhibit CCEs in a characteristic, age-dependent pattern (23-26). In the R1.40 AD model (27-29), neuronal CCEs occur approximately 6 months prior to the first appearance of $A \beta$ deposits, but lowering or eliminating $A \beta$ production via genetic means in the R1.40 mouse model causes either a delay or a complete block of neuronal CCEs (30).

The current studies were designed to explore the A $\beta$-dependent pathways responsible for induction of neuronal CCEs. We demonstrated that alterations in brain microglia were coincident with the first evidence of neuronal CCEs in the R1.40 model and that the microglial alterations were dependent on $\mathrm{A} \beta$ generation. Induction of systemic inflammation promoted the early appearance of neuronal CCEs in young R1.40 animals, but not in nontransgenic controls. In addition, inhibition of neuroinflammation in young R1.40 animals by chronic administration of 2 commonly used

\section{Figure 1}

A $\beta$-dependent alterations in brain microglia in R1.40 transgenic mice. (A and B) Neocortical microglia in 6-month-old nontransgenic mice exhibited extensive fine processes with small cell bodies. (C and $\mathbf{D}$ ) Age-matched R1.40 animals exhibited reactive neocortical microglia: thick, asymmetrically oriented processes surrounding a swollen cell body. (E and F) R1.40;Bace1--- animals at 6 months of age exhibited microglia with a resting morphology that was indistinguishable from that of nontransgenic controls ( $\mathbf{A}$ and $\mathbf{B})$. ( $\mathbf{G}$ and $\mathbf{H}) \mathbf{C} \times 3 c r 1^{+/ g f p}$ mice lacking the R1.40 transgene (G) displayed GFP-expressing microglia morphologically similar to the Iba1-stained microglia observed in nontransgenic controls (A and B). Conversely, Cx3cr1+/gfp mice transgenic mice with the $\mathrm{R} 1.40$ transgene $(\mathbf{H})$ exhibited microglia with a reactive morphology similar to that of 6-month-old R1.40 transgenic mice (C and D). (I) Morphometric analysis of Iba1-positive microglia revealed significantly higher FF values in R1.40 animals than in nontransgenic (nTg) and R1.40;Bace1-/- mice. ${ }^{\star} P<0.01 ;{ }^{* *} P<0.0004$. Scale bars: $500 \mu \mathrm{m}(\mathbf{A}, \mathbf{C}$, and E); $100 \mu \mathrm{m}$ (B, D, F, G, and H).

NSAIDs, ibuprofen and naproxen, blocked alterations in brain microglia as well as neuronal CCEs in the absence of detectable alterations in APP processing and A $\beta$ metabolism. Notably, therapeutic NSAID treatment of older R1.40 mice did not reverse the presence of extant CCEs, even after several months of treatment. These results argue for a role of both $A \beta$ and neuroinflammation in the induction of neuronal CCEs and provide a potential explanation for the relative successes and failures of the retrospective and prospective NSAID trials in the treatment of human AD.

\section{Results}

Alterations in brain microglia in the R1.40 mouse model of $A D$. To determine whether the induction of neuronal CCEs was accompanied by alterations in brain microglia, we used the genomic-based R1.40 transgenic mouse model of $\mathrm{AD}$, which contains the entire Swedish mutant human APP (see Methods). Because this model is under endogenous human regulatory elements, it mimics the correct temporal and spatial expression patterns observed in human brain tissue. The R1.40 mouse, when maintained on the C57BL/6 inbred genetic background, first begins to exhibit $\mathrm{A} \beta$ deposition and neuritic abnormalities at $12-14$ months of age (31). In addition, the R1.40 mouse model exhibits neuronal CCEs in many of the same brain regions in which they are encountered in human $\operatorname{AD}(23,30)$.

Brain sections of adult R1.40 and control mice were examined for the microglial marker Iba 1 via immunohistochemistry at a variety of ages. R1.40 mice exhibited microglial activation and migration to sites of neocortical $A \beta$ deposition beginning at about 12-14 months of age. Unexpectedly, however, immunohistochemical analysis of microglial morphology in 6-month-old R1.40 animals also demonstrated noticeable alterations (Figure 1, C and D). In 6-month-old nontransgenic animals, microglia displayed a resting phenotype, with numerous thin branching processes extending away from a small cell body (Figure 1, A and B). Conversely, R1.40 microglia exhibited shorter, asymmetrically oriented processes as well as swollen cell bodies (Figure 1, C and D). None of these changes was evident at 4 months of age in R1.40 animals (data not shown), which suggests that, similar to induction of neuronal CCEs, alterations in brain microglia occur between 4 and 6 months of age. Notably, the microglia with altered morphologies in the R1.40 mice were not positive for CD45, a cell surface protein expressed in highly activated microglia as well as in infiltrating immune cells (data not shown). 


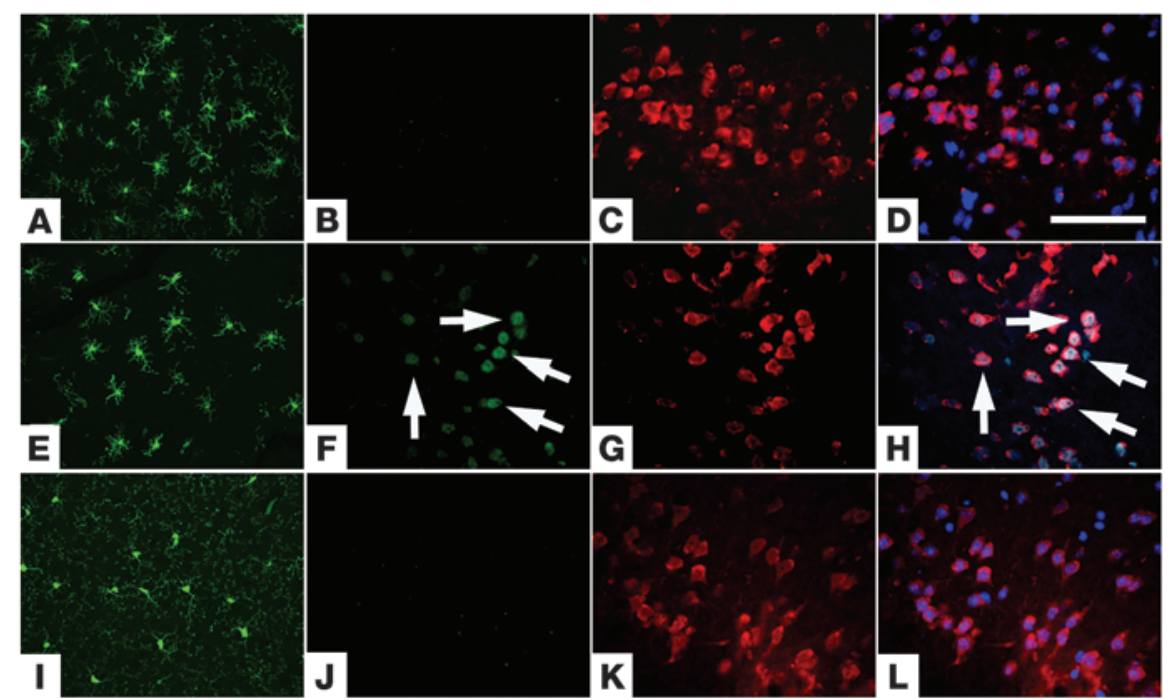

Figure 2

LPS administration provokes neuroinflammation and neuronal CCEs. (A-D) Nontransgenic mice at 2 months of age subject to LPS injections exhibited lba1-immunoreactive neocortical microglia with an activated morphology $(\mathbf{A})$ with no evidence of expression of cyclin $D(\mathbf{B})$ in NeuN-positive neurons (C). (E-H) Age-matched R1.40 transgenic animals injected with LPS exhibited Iba1-positive microglia with an activated morphology (E) as well as expression of cyclin $D(\mathbf{F})$ in a subset of NeuN-positive cortical layer II/III neurons $(\mathbf{G})$. (I-K) Age-matched R1.40 animals injected with PBS exhibited lba1-positive microglia with a resting morphology (I) and no evidence of expression of cyclin D (J) in NeuN-positive neurons (K). Similar results were obtained with immunohistochemistry for the cell cycle protein cyclin A (not shown). (D, H, and $\mathbf{L}$ ) Merged images. Nuclei were counterstained with DAPI (blue). Arrows indicate cyclin Dpositive neurons. Scale bar: $100 \mu \mathrm{m}$.

In order to confirm these observations, R1.40 transgenic mice were crossed to a mouse line in which the Gfp gene is knocked into the fractalkine receptor locus $(\mathrm{C} x 3 \mathrm{cr} 1)$, leading to the specific labeling of all brain microglia with GFP. We generated mice heterozygous for the $C \times 3 \mathrm{cr} 1$ knockin $\left(\mathrm{C} \times 3 \mathrm{cr} 1^{+/ g f p}\right)$ with and without the R1.40 transgene and examined their brain sections via fluorescent confocal microscopy. Similar to the immunohistochemical data, GFP in the microglia of 6-month-old $\mathrm{C} \times 3 \mathrm{cr} 1^{+/ g f p}$ mice lacking the R1.40 transgene exhibited a resting phenotype, with numerous thin, branching processes (Figure $1 \mathrm{G}$ ). However, microglia in $\mathrm{C} \times 3 \mathrm{cr} 1^{+/ g f p}$ mice with the R1.40 transgene exhibited a more activated phenotype, including shortened processes and enlarged cell bodies (Figure 1H). To obtain a quantitative measure of microglial activation, morphometric analysis of brain microglia was conducted using form factor (FF; see Methods). In general, a higher FF value is indicative of more bushy, activated microglia, while a lower FF value is indicative of resting microglia (32-34). Notably, FF analysis demonstrated that Iba1-positive microglia in 6-monthold R1.40 animals had a significantly higher mean FF value than did microglia in age-matched nontransgenic controls (Figure 1I).

To specifically test whether $\mathrm{A} \beta$ generation from the R1.40 transgene is required for the alterations in brain microglia observed at 6 months of age, R1.40 transgenic mice were mated with animals lacking $A \beta$ precursor protein cleavage enzyme 1 (Bace1), the primary $\beta$-secretase required for the first step in the generation of $A \beta$ from APP. Iba1 immunostaining of cortical microglia in 6-monthold R1.40 transgenic mice lacking Bace1 (R1.40;Bace $1^{-/-}$mice) exhibited a resting phenotype (Figure $1, \mathrm{E}$ and $\mathrm{F}$ ) resembling that of age-matched nontransgenic controls (Figure 1, A and B). These double-mutant microglia had FF values that were significantly lower than those of R1.40 animals, but indistinguishable from those of agematched nontransgenic animals (Figure 1I). Similarly, analysis of fractal dimension, another morphometric parameter inversely correlated with microglial activation state (32-34), was lower in 6-month-old R1.40 mice than in R1.40;Bace $1^{-/-}$mice or nontransgenic controls (data not shown). Together with our previous data demonstrating that the induction of neuronal CCEs at 6 months of age is also dependent on the amyloidogenic processing of APP in the R1.40 mouse model (30), these results suggest that alterations in microglial activation could play a direct role in the $A \beta$ dependent induction of neuronal CCEs.

Induction of inflammation promotes microglial activation and neuronal CCEs. Based on the correlative data linking microglial alterations and neuronal CCEs, we next examined whether induction of inflammation is capable of inducing premature neuronal CCEs in young R1.40 animals. We intraperitoneally injected 2 -month-old nontransgenic and R1.40 mice with either LPS (20 $\mu \mathrm{g}$ /animal once daily for 4 days) or PBS. This LPS injection protocol has previously been demonstrated to induce microglia activation within the brain (35). As expected, LPS exposure induced robust microglial activation in both R1.40 mice (Figure 2E) and nontransgenic controls (Figure 2A) compared with animals injected with PBS alone (Figure 2I). Additional sections from these animals were stained for cell cycle proteins and the neuronal marker NeuN. PBS-injected R1.40 animals did not exhibit any evidence of cyclin D-positive neurons in frontal cortical layers II/III (Figure 2, J-L). Conversely, LPS-injected R1.40 animals exhibited numerous cyclin D-positive neurons in the same brain regions (Figure 2, F-H). Staining for cyclin A gave similar results (data not shown). Notably, LPS-injected nontransgenic controls exhibited no evidence of neuronal CCEs (Figure 2, B-D). Taken together, these data suggest that the induction of neuronal CCEs is dependent on both induction of inflammatory processes and an $\mathrm{R} 1.40$ genotype, and thus, most likely, on $\mathrm{A} \beta$ generation.

Early NSAID treatment inhibits inflammatory responses and neuronal CCEs. To determine whether inhibition of the microglial alterations in R1.40 transgenic mice blocks induction of neuronal CCEs, we placed 3-month-old R1.40 and aged-matched nontransgenic controls on standard laboratory diets with or without ibuprofen or naproxen (375 ppm in chow) for 3 months. At 6 months of age, Iba1 immunohistochemistry of treated R1.40 animals revealed microglial cells with thin, symmetric processes, indicating that both ibuprofen and naproxen (Figure 3, C-F) blocked the microglial activation (Figure 3, A and B) to levels resembling the resting phenotype exhibited by age-matched nontransgenic controls (Figure 1, A and B). Morphometric analysis of Iba1-positive brain microglia lent support to these conclusions, as FF was substan- 

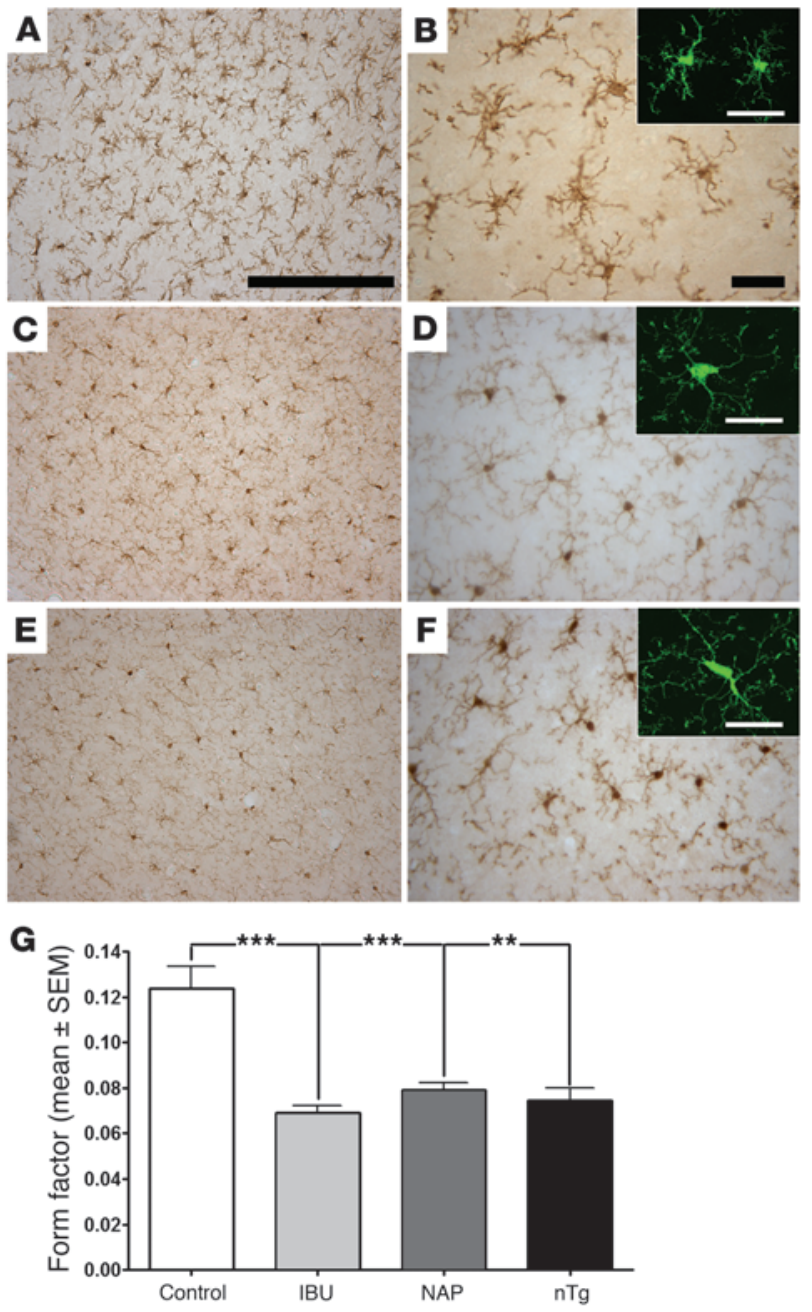

tially reduced in the ibuprofen and naproxen treatment groups compared with untreated R1.40 mice and was indistinguishable from that of age-matched nontransgenic controls (Figure $3 \mathrm{G}$ ).

Brain sections from the control and NSAID treatment groups were also examined for alterations in neuronal CCEs. As previously reported (30), by 6 months, R1.40 transgenic mice exhibited a substantial number of neurons positive for cyclin A (Figure $4, \mathrm{~A}-\mathrm{C}$ ) and cyclin D (data not shown) in layers II/III of frontal cortex. These same neuronal populations also exhibited evidence of DNA replication, as indicated by 3 or 4 spots of hybridization with a DNA FISH probe for either mouse chromosome 16 (Figure $4 \mathrm{C}$, inset) or the $\mathrm{R} 1.40$ human transgene on mouse chromosome 13 (data not shown). Administration of ibuprofen or naproxen blocked virtually all evidence of CCEs, as measured by cyclin A and cyclin D expression (Figure 4, D-I) as well as by DNA replication (Figure 4, F and I, insets). Taken together, these data indicate that a 3-month treatment of young R1.40 mice with either ibuprofen or naproxen blocks both microglial activation and development of neuronal CCEs in the mouse 6 months prior to the initiation of $A \beta$ deposition in this model.

The effectiveness of the treatments was quantified by determining the percentage of neurons in frontal cortex layers II/III positive for cyclin A and cyclin D as well as the percentage exhibiting a hyperploid phenotype with a DNA probe specific to mouse chro-

\section{Figure 3}

Prevention trial of NSAIDs inhibits microglial alterations. (A and B) R1.40 transgenic mice placed on a control diet for 3 months beginning at 3 months of age exhibited lba1-immunoreactive neocortical microglia with an activated phenotype, similar to R1.40 animals at 6 months of age (see Figure 1, C and D). (C-F) R1.40 animals placed on a 3-month ibuprofen- (C and $\mathbf{D}$ ) or naproxen-containing ( $\mathbf{E}$ and $\mathbf{F}$ ) diet exhibited lba1-positive microglia with a resting phenotype resembling that of 6-month-old nontransgenic control and R1.40;Bace 1-/animals (see Figure 1, A, B, E, and F). Insets show representative confocal images of Iba1-positive microglia stained with fluorescently tagged secondary antibodies. (G) Morphometric analysis of Iba1-positive microglia revealed significantly lower FF values in mice treated with ibuprofen (IBU) and naproxen (NAP), compared with R1.40 animals fed a control diet, that were not different from the FF values in nontransgenic mice. ${ }^{* \star} P<0.0004 ;{ }^{* \star \star} P<0.0002$. Scale bars: $500 \mu \mathrm{m}$ (A, C, and E); $100 \mu \mathrm{m}$ (B, D, F, and insets).

mosome 16 or the R1.40 transgene on chromosome 13 (Figure 5 , A-D). In 6-month-old R1.40 transgenic mice, $43 \%$ of the neurons (NeuN-positive cells) in cortical layers II/III were positive for cyclin A (Figure 5A). NSAID treatment for 3 months with ibuprofen reduced the percentage of cyclin A-positive neurons in cortical layers II/III to approximately $4 \%$, whereas naproxen reduced it to about 3\%. Cyclin D expression was reduced to approximately $5 \%$ and $3 \%$ by ibuprofen and naproxen, respectively (Figure $5 \mathrm{~B}$ ). These levels were statistically indistinguishable from those of agematched nontransgenic controls (cyclin A, $0.9 \%$; cyclin D, $1.4 \%$ ).

Quantitative analysis of the percentage of hyperploid neuronal nuclei in cortical layers II/III was determined after FISH with a probes specific for R1.40 mouse chromosome 16 or chromosome 13. Untreated R1.40 transgenic mice exhibited hyperploidy for both probes: $12 \%$ for chromosome 16 (Figure 5C) and 9\% for chromosome 13 (Figure 5D). After ibuprofen and naproxen treatment, these percentages were reduced to $0.6 \%$ and $0.4 \%$, respectively. These levels were statistically indistinguishable from those found in nontransgenic controls.

Lack of effect of chronic NSAID treatment on APP processing and A $\beta$ metabolism. Considerable evidence suggests that NSAIDs can act as GSMs in acute treatment paradigms both in vitro and in vivo, but their potency varies widely. In these short-term assays, ibuprofen is reported to be most effective, while naproxen lacks any apparent GSM activity $(14,15)$. This difference guided our choice of ibuprofen and naproxen for the current studies as a way of distinguishing whether any effects we observed were caused by differences in $A \beta$ generation. Having found no difference in their ability to inhibit both microglial activation and CCE induction, we next analyzed our samples to determine whether the impact of 3 months of chronic NSAID administration differs from the short-term effects reported previously $(14,15)$. Brain extracts from 6-month-old treated and untreated animals were analyzed via Western blot for levels of holo-APP and APP C-terminal fragments (CTFs). No significant differences in the steady-state levels of holo-APP were observed in samples from either NSAID treatment group compared with controls (Figure 6, A and B). Western blot analysis also revealed that the levels of the 2 primary APP CTFs, CTF $\alpha$ and CTF $\beta$, were unchanged (Figure 6A). A $\beta$ metabolism was similarly unaffected. Finally, the steady-state levels of both major isoforms of $A \beta, A \beta_{1-40}$ and $A \beta_{1-42}$, were determined by ELISAs from formic acid-treated brain extracts. Neither ibuprofen nor naproxen treatment led to significant alterations in 

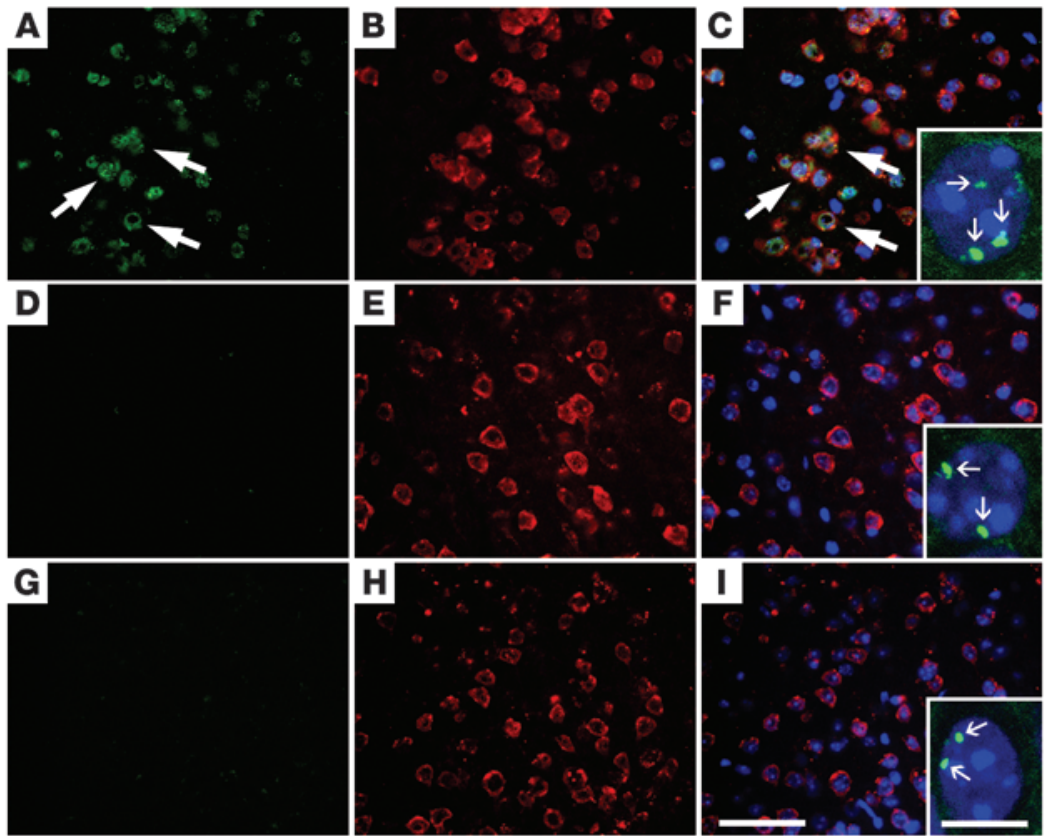

\section{Figure 4}

Prevention trial of NSAIDs inhibits neuronal CCEs. (A-C) R1.40 transgenic mice placed on a control diet for 3 months beginning at 3 months of age exhibited expression of cyclin $\mathbf{A}(\mathbf{A})$ in numerous NeuN-positive (B) neurons on the frontal cortex layers II/III, similar to previously published results (30). Large arrows indicate cyclin A-positive neurons. (D-I) R1.40 animals placed on the 3-month ibuprofen- (D-F) or naproxen-containing $(\mathbf{G}-\mathbf{I})$ diet exhibited expression of cyclin A ( $\mathbf{D}$ and $\mathbf{G}$ ) in a subset of NeuN-positive ( $\mathbf{E}$ and $\mathbf{H}$ ) neurons. Similar results were obtained with immunohistochemistry for the cell cycle protein cyclin $D$ (not shown). (C, F and I) Merged images. Nuclei were counterstained with DAPI (blue). FISH with a DNA probe to mouse chromosome 13 demonstrated a subset of neurons with 3 or 4 spots of hybridization in $\mathrm{R} 1.40$ animals fed the control diet (C, inset), while the ibuprofen and naproxen treatment groups exhibited only 2 spots of hybridization ( $\mathbf{F}$ and $\mathbf{I}$, insets). Small arrows indicate FISH signals in the neuronal nucleus. Scale bars: $100 \mu \mathrm{m}$ (A-I); $10 \mu \mathrm{m}$ (insets). the steady-state levels of $A \beta_{1-40}$ or $A \beta_{1-42}$ compared with untreated transgenic controls (Table 1 ). These results suggest that chronic administration of NSAIDs blocks microglial activation and neuronal CCEs by a mechanism independent of their effect on $\mathrm{APP}$ processing and $\mathrm{A} \beta$ metabolism.

NSAID treatment cannot reverse previously established neuronal CCEs. Neuronal CCEs develop in a precise temporal and spatial pattern in R1.40 mice (30). Neurons in frontal cortical layers II/III first exhibited CCEs at 6 months of age, whereas neurons in cortical layers V/VI did not exhibit CCEs until 12 months of age (Figure 7A). Furthermore, after a specific brain region develops CCEs, the percentage of affected cells remains stable over the lifespan of the animal (30). This predictable time sequence offered us the opportunity to determine whether NSAID administration can reverse the accumulation of neuronal CCEs in cortical layers II/III after they begin to form. R1.40 transgenic mice were placed on control, ibuprofen-containing, or naproxen-containing diets (375 ppm in each) for 6 months beginning at 6 months of age. Brain sections from the 12-month-old animals were subsequently examined for the presence of neuronal CCEs via immunohistochemistry and FISH. Immunohistochemistry for cyclin D and FISH revealed the presence of numerous immunopositive layer II/III neurons in control animals as well as in animals treated with ibuprofen or naproxen (Figure 7, B-G).

Quantification of the data confirmed the visual impressions from this neuronal population. Similar to the results obtained at 6 months of age (Figure 5), $45 \%-50 \%$ of neurons within frontal cortical layers II/III were positive for cyclin A and cyclin D in 12-monthold R1.40 transgenic mice (Figure 8, A and B). In addition, the number of neurons with 3 or 4 spots of hybridization in frontal cortical layers II/III remained unchanged between 6 and 12 months of age: $10 \%-15 \%$ hyperploidy for both chromosome 16 and chromosome 13

\section{Figure 5}

Quantification of inhibition of neuronal CCEs in prevention trial of NSAIDs. Percentages of NeuN-positive neurons in cortical layers II/III exhibiting expression of cyclin A (A) or cyclin D (B) as well as 3 or 4 spots of hybridization with FISH for DNA probes from mouse chromosome 16 (C) and 13 (D) was calculated in R1.40 transgenic mice fed control, ibuprofencontaining, and naproxen-containing diets as well as in nontransgenic mice fed the control diet $(n=5) .{ }^{\star *} P<0.003,{ }^{* \star \star} P<0.001$ versus control diet-fed R1.40 transgenic mice.
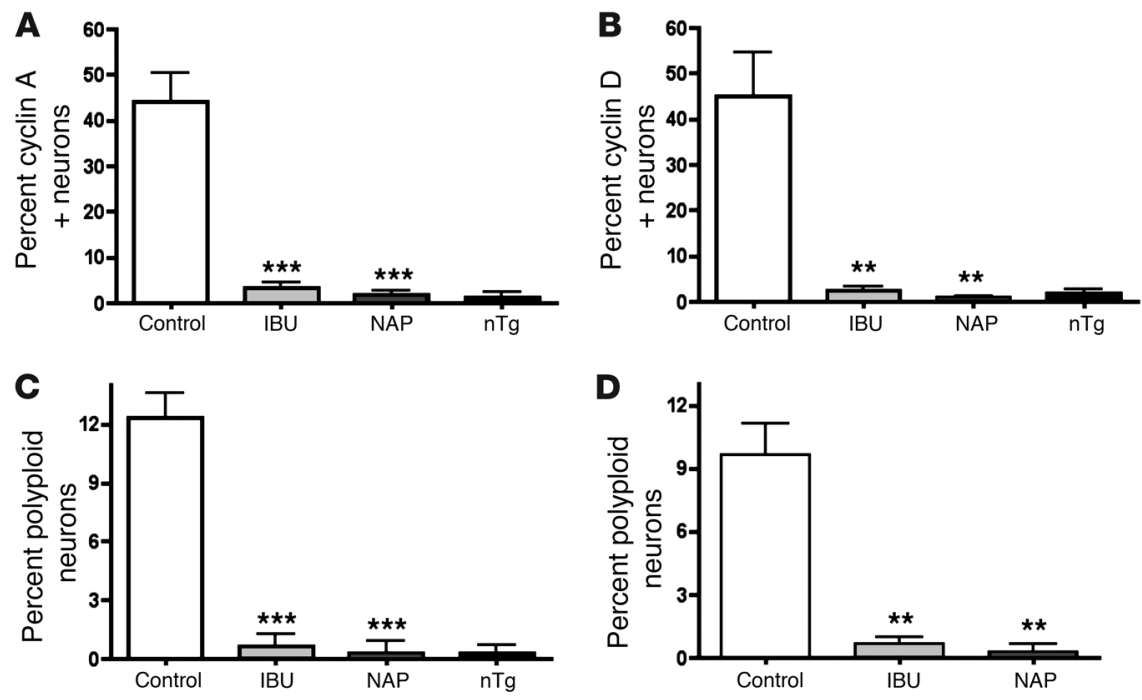
A
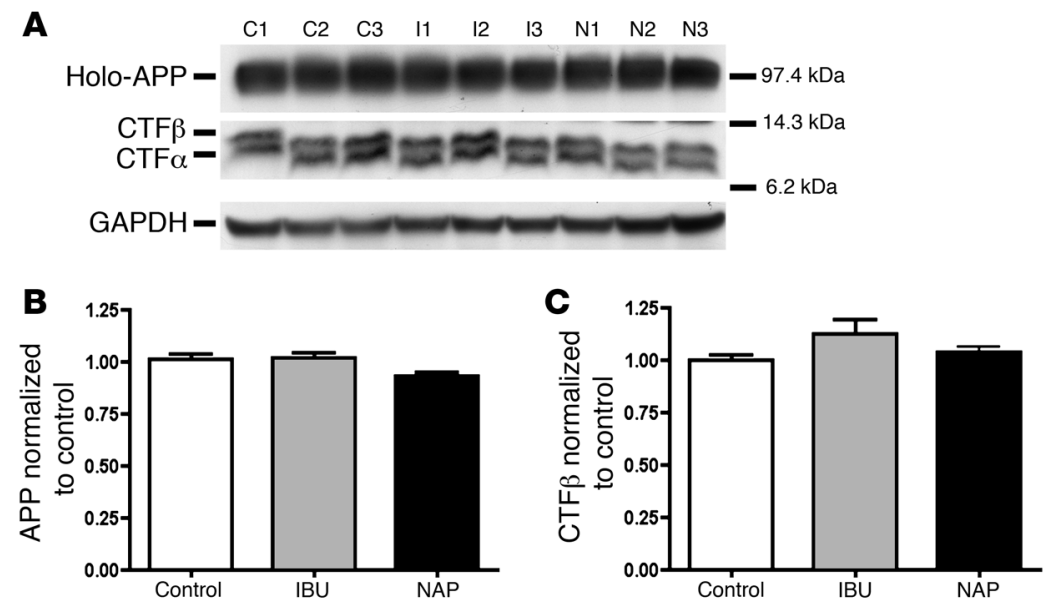

\section{Figure 6}

Lack of effect of chronic NSAID treatments on APP processing. (A) Western blots of brain extracts from the R1.40 animals fed control (C1-C3), ibuprofen-containing (I1-I3), and naproxen-containing (N1-N3) diets were probed with antibodies to the $\mathrm{C}$ terminus of APP and subsequently stripped and reprobed with an antibody against GAPDH as a loading control. Shown on the right is the approximate size in $\mathrm{kDa}$. (B and $\mathbf{C}$ ) Relative levels of holo-APP (B) and CTF $\beta$ (C) were quantified $(n=3)$ from animals on the control, ibuprofen-containing, and naproxen-containing diets by normalizing the intensity values of APP and APP CTF $\beta$ to GAPDH. No significant differences were observed in the relative levels of holoAPP or CTF $\beta$ between treatment groups.
(Figure 8, C and D). Although DNA replication is not reversible, we were surprised to find that after 6 months of treatment with either ibuprofen or naproxen, there was no significant reduction in the percentage of cyclin A- or cyclin D-positive neurons (Figure $8, A$ and $B$ ). These data suggest that NSAID administration in a therapeutic regimen (after the disease has initiated) is incapable of reversing the previously established neuronal CCEs.

Late NSAID treatment can block the development of additional neuronal CCEs. Neuronal CCEs first appeared in cortical layers V/VI at 12 months of age in the R1.40 mice (Figure $7 \mathrm{H}$ ). To determine whether a 6-month treatment with ibuprofen or naproxen could block CCEs in this neuronal population, we turned our attention to the deeper cortical layers of our animals. Unlike the results obtained for the existing CCEs in frontal cortex layers II/III (Figure 7, B-G), both ibuprofen and naproxen led to a dramatic reduction in the number of cyclin A- and cyclin D-positive neurons in layers V/VI at 12 months of age (Figure 7, J-M). FISH analysis with probes from mouse chromosome 13 (Figure 7, I, K, and $\mathrm{M}$, insets) and chromosome 16 (data not shown) also revealed a decrease in the number of hyperploid neuronal nuclei. Quantitative analysis revealed that approximately $50 \%$ of the NeuN-positive cells in cortical layers V/VI were positive for cyclin A and cyclin D in untreated transgenic mice (Figure 8, A and B). After treatment with ibuprofen or naproxen, however, there was a significant reduction in the percentage of cortical layer V/VI neurons expressing cell cycle proteins. Quantitative analysis of the percentage of hyperploid neuronal nuclei by FISH with DNA probes for mouse chromosome 16 and chromosome 13 revealed a similar picture (Figure 8, C and D). Thus, long-term administration of NSAIDs after neuronal CCEs was capable of blocking subsequent events, but could not reverse the damage in previously affected cells.

\section{Discussion}

There is increasing evidence that the neurodegeneration observed in $\mathrm{AD}$ is the result of a pathogenic process initiated well before the onset of the cognitive symptoms associated with the disease. Although biochemical, genetic, and pathological studies clearly implicate alterations in APP processing as being central to $\mathrm{AD}$ pathogenesis, the relationship between $\mathrm{A} \beta$ generation and deposition and the other features of the disease remains unclear. For example, both cross-sectional pathological studies and PET studies with the amyloid binding Pittsburgh compound B have revealed that a relatively large number of aged, cognitively normal individuals have a substantial amyloid plaque load (36-39). This has direct implications for both the study of pathogenic mechanisms underlying $\mathrm{AD}$ and the various prospective clinical trials being conducted in human AD.

Our previous studies demonstrated that one of the most reliable indicators of neuronal populations at risk in $\mathrm{AD}$ is ectopic expression of cell cycle proteins and DNA replication in postmitotic neurons (18-21). CCEs identify neurons at risk in AD as well as in mild cognitive impairment, which suggests that they represent an early marker of neuronal vulnerability $(21,40,41)$. Their involvement in the core biology of $\mathrm{AD}$ is further documented by their presence in most transgenic mouse models of $\mathrm{AD}$ that have been examined. In R1.40 animals, ectopic CCEs first appear 6-8 months prior to A $\beta$ deposition and subsequently advance in a precise temporal and spatial pattern in populations of neurons identical to those at risk in the human disease (23). In addition, our previous studies demonstrated that the appearance of neuronal CCEs is $\beta$-secretase dependent (30).

The current studies expand these insights in several ways. Although late-stage markers of microglial activation do not develop until later in disease progression (31), cortical microglia underwent visible morphological changes between 4 and 6 months of age in R1.40 mice, coincident with the first appearance of neuronal CCEs. This evidence of microglial activation was dependent on $A \beta$ generation, as it was greatly reduced in R1.40 animals lacking Bace1. Although alterations in microglia are typically reported to be elicited in response to the deposition of $A \beta$, several recent findings suggest that alterations in microglia as well as in the production of cytokines and chemokines may be an early feature that precedes $A \beta$ deposition in other mouse

\section{Table 1}

Chronic dosing with NSAIDs does not alter steady-state brain A $\beta$ levels

\begin{tabular}{lcccc} 
Treatment & $\boldsymbol{n}$ & $\mathbf{A} \beta_{1-40}\left(\mathbf{p m o l} \cdot \mathbf{g}^{-1}\right)$ & $\mathbf{A} \beta_{1-42}\left(\mathbf{p m o l} \cdot \mathbf{g}^{-1}\right)$ & $\mathbf{A} \beta_{1-42} / \mathbf{A} \beta_{1-40}$ \\
Control & 10 & $8.98(1.86)$ & $3.36(0.71)$ & $0.39(0.11)$ \\
Ibuprofen & 10 & $9.00(1.23)$ & $3.09(0.48)$ & $0.35(0.05)$ \\
Naproxen & 10 & $10.57(2.97)$ & $3.15(0.71)$ & $0.33(0.11)$ \\
\hline
\end{tabular}

B6-R1.40 mice were dosed as described in the text. Values are expressed as mean (SD). 
A

Layers II/III

Layers V/VI

\begin{tabular}{l|l}
\hline \\
0
\end{tabular}

Layer II/III
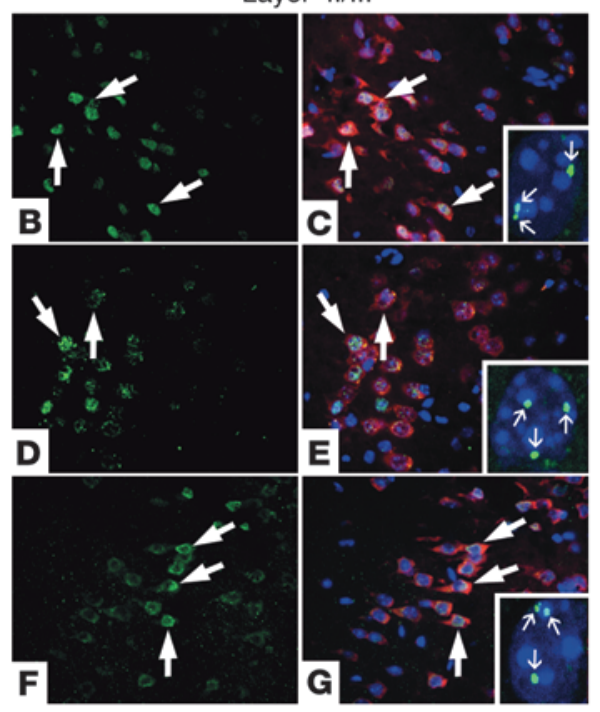

12 Neuronal cell cycle events 24

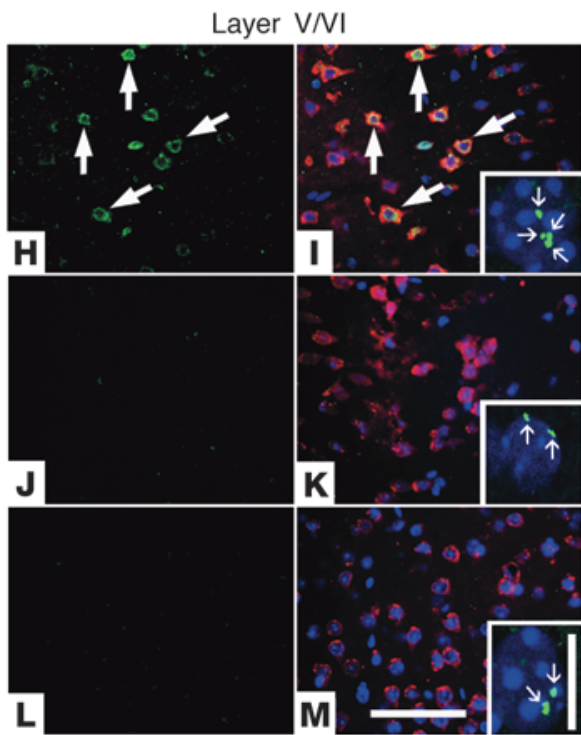

Figure 7

Therapeutic trial of NSAIDs inhibits subsequent, but not extant, neuronal CCEs. (A) Neuronal CCEs were first observed in frontal cortical layers II/III at 6 months of age and persisted for 2 or more years in the R1.40 animals. Neuronal CCEs were not observed in deeper cortical layers V/VI until 12 months of age. (B-M) R1.40 transgenic mice at 6 months of age were fed control (B, $\mathbf{C}, \mathbf{H}$, and I), ibuprofen-containing (D, E, J, and $\mathbf{K})$ or naproxen-containing $(\mathbf{F}, \mathbf{G}, \mathbf{L}$, and $\mathbf{M})$ diets for 6 months. (B and $\mathbf{H}$ ) Control diet-fed mice exhibited expression of cyclin $\mathbf{D}$ (large arrows) in NeuN-positive neurons in frontal cortex layers II/III and layers V/VI. (D, F, J, and L) Ibuprofen- or naproxen-containing diet-fed mice exhibited expression of cyclin $D$ in a subset of NeuN-positive neurons in layers II/III ( $\mathbf{D}$ and $\mathbf{F}$ ), with minimal expression of cyclin D in layers $\mathrm{V} / \mathrm{VI}$ ( $\mathbf{J}$ and $\mathbf{L})$. (C, E, G, I, K, and M) Merged images. Sections were stained with NeuN (red), and nuclei were counterstained with DAPI (blue). FISH analysis with a DNA probe specific for mouse chromosome 16 demonstrated the presence of a subset of neuronal nuclei with 3 or 4 spots of hybridization (small arrows) in all treatment groups in cortical layers II/II (C, E, and G, insets) and only the control diet group in cortical layers V/VI (I, K, and M, insets). Scale bars: $100 \mu \mathrm{m}$ (B-M); $10 \mu \mathrm{m}$ (insets).
Age (mo) 0

eliminate the appearance of neuronal CCEs. Treatment of R1.40 mice with ibuprofen or naproxen from 3-6 months of age represented the murine equivalent of a prevention trial. In this situation, in which therapy was initiated 3 months prior to the first appearance of neuronal CCEs, both ibuprofen and naproxen were capable of reducing the microglial morphological alterations and the appearance of CCEs normally observed at 6 months of age. Our biochemical analysis suggests that this effect is independent of any significant chronic change in APP processing.

Although this prevention paradigm holds out great hope for clinical application, the NSAID therapeutic trial is a sobering reminder of the complexity of the disease. NSAID treatment begun after the first appearance of neuronal CCEs could prevent the appearance of neuronal CCEs that normally appear in the deeper cortical layers V/VI at 12 months of age, but this treatment was incapable of reversing the neuronal CCEs already present in cortical layers II/III. The failure to reduce the levels of cell cycle proteins in these animals is especially noteworthy. It would be expected that the long period of drug treatment should substantially reduce the inflammation caused by the APP transgene and its A $\beta$ byproduct. Despite this, 6 months of therapy (one-fifth to one-quarter of a mouse lifetime) was incapable of restoring the normal cell cycle protein status of the affected neurons.

There are considerable data regarding the efficacy of NSAIDs in the prevention and/or treatment of AD. Retmodels of $\mathrm{AD}(42,43)$. In contrast to recent results suggesting that peripheral immune cells may play a role in $A \beta$ metabolism (44-46), we think that this is unlikely to be a major contributor to our present results, as the Iba1-positive microglia in the R1.40 mice did not stain with CD45, a cell surface protein expressed at high levels on infiltrating monocytes (data not shown).

This early microglial activation may well play a causative role in the appearance of neuronal CCEs. We have demonstrated this in both positive and negative directions. When mice were subjected to an inflammatory challenge independent of that triggered by $\mathrm{A} \beta$ itself, the appearance of cell cycle changes could be advanced by months. Significantly, although both nontransgenic and R1.40 mice exhibited robust microglial activation, only the genetically susceptible R1.40 transgenic mice went on to reveal neuronal CCEs. These observations imply that both conditions - genetics and inflammation - must be met to produce neuronal cell cycling.

The causative nature of the inflammatory process was further suggested by our observation that NSAID treatment could virtually rospective epidemiological studies suggest that a wide variety of NSAIDs may significantly reduce one's lifetime risk of developing $\mathrm{AD}(6)$, although the effect may not be felt rapidly (8). Long-term use of NSAIDs is associated with protection from the development of $\mathrm{AD}(47)$ and reduction in $\mathrm{A} \beta$ deposition in mouse models of $\mathrm{AD}$ $(10,11,48,49)$ and is also positively correlated with a reduction of plaque-associated microglia in both humans $(50,51)$ and mice $(10,11,48,49)$. Inflammatory events found in mouse models of $\mathrm{AD}$ - namely, microglial activation and expression of proinflammatory molecules - are significantly reduced by NSAID treatment $(11,48,49,52,53)$. Yet in opposition to these findings of efficacy are the disappointing outcomes of prospective trials with NSAIDs in subjects with mild to moderate AD. No detectable effects on a variety of clinical outcome measures of $\mathrm{AD}$ progression were found in controlled trials of naproxen, celecoxib, and rofecoxib (9, 54). Indeed, a recent trial of more than 2,500 individuals reported no significant cognitive improvement after 4 years of treatment with either naproxen or celecoxib (9). In addition, a large phase III 

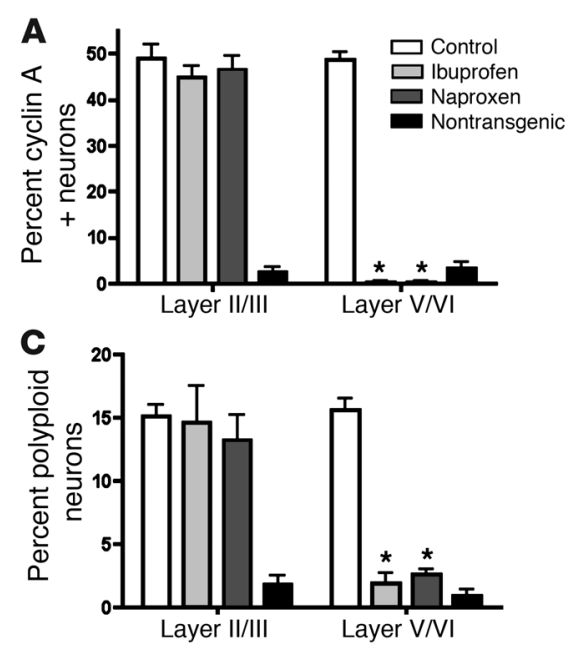
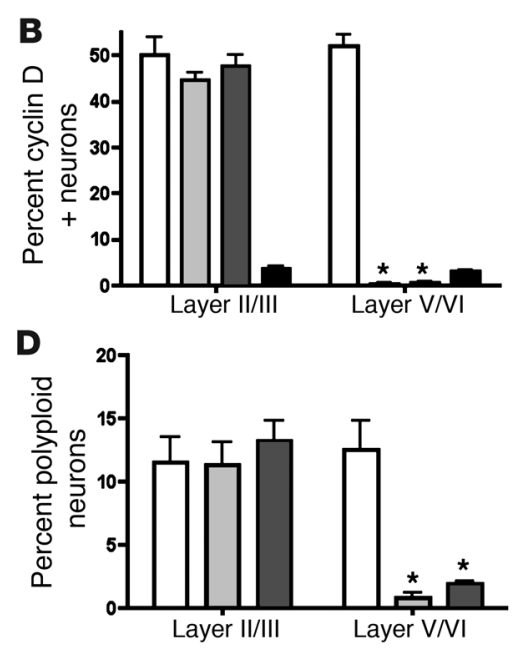

\section{Figure 8}

Quantification of inhibition of neuronal CCEs in NSAID therapeutic trial. Percentages of NeuN-positive neurons in cortical layers II/III and layers V/VI exhibiting expression of cyclin $\mathbf{A}(\mathbf{A})$ or cyclin $(\mathbf{B})$ as well as 3 or 4 spots of hybridization with FISH for DNA probes from mouse chromosome 16 (C) and 13 (D) were calculated in R1.40 transgenic mice fed control, ibuprofen-containing, and naproxencontaining diets as well as in nontransgenic mice fed the control diet $(n=5)$. ${ }^{*} P<0.001$ versus control diet-fed R1.40 transgenic mice. clinical trial of flurbiprofen was recently completed without any evidence of cognitive improvement in $\mathrm{AD}$ subjects (55). The inability of NSAID treatment of R1.40 mice to reverse CCEs once they begin to appear is consistent with the human trial data and suggests that, unlike initiation, the progression of $\mathrm{AD}$ may be independent of the presence of inflammation in the brain.

It must be considered that the NSAIDs are acting in multiple different ways in these studies. First, the NSAIDs may act to block microglial activation via inhibition of cyclooxygenase directly in the brain, a mechanism that is supported by our present findings. Second, NSAIDs are well recognized to also inhibit cyclooxygenase in the systemic immune system, and this could have secondary consequences for altered infiltration and/or activation of peripheral immune cells within the brain. Although we cannot rule this out, Iba1-positive microglia in 6-month-old R1.40 mice (with or without NSAIDs) did not stain with CD45 (data not shown). Third, NSAIDs may have off-target effects. Some studies have demonstrated that an acute, relatively shortterm administration of selected NSAIDs to AD mouse models results in decreased production of longer $A \beta$ peptides, in favor of shorter, less-amyloidogenic isoforms (56), although not all studies have observed this effect (57). This would offer a potential nonimmune mechanism of NSAID action.

Unlike these previous reports, we found that chronic NSAID treatment of predepositing R1.40 mice resulted in no significant alterations in the steady-state levels of $A \beta_{1-40}, A \beta_{1-42}$ or in their ratio. Many factors might explain these differences; we believe the main factor is the long-term nature of our trials in animals that are predepositing. Most of the chronic NSAID studies in mouse models of $\mathrm{AD}$ have focused on $\mathrm{A} \beta$ deposition as the primary outcome measure, which is supported by our recent finding of reduced $A \beta$ deposition in the R1.40 mouse model of AD when treated with 375 ppm ibuprofen from 15 to 24 months of age (N.H. Varvel, G.E. Landreth, B.T. Lamb, and K. Herrup, unpublished observations). Our data are also consistent with recent studies demonstrating that treatment of the Tg2576 mouse model of AD with identical doses of ibuprofen and naproxen for 30 days in predepositing animals does not alter $\mathrm{A} \beta$ levels (48).

It has also been suggested that NSAIDs regulate Bace 1 expression levels through a PPAR $\gamma$-mediated pathway. Certain NSAIDs, including ibuprofen, are agonists for PPAR $\gamma$ (58). Activation of
PPAR $\gamma$ with the selective agonist pioglitazone reduces Bace1 levels as well as steady-state levels of CTF $\beta$ in a mouse model of AD (17), but any direct relationship between ibuprofen administration and suppression of Bace1 levels remains unresolved. In our studies, we found no differences in CTF $\beta$ levels between the NSAID treatment groups, confirming our conclusion that the primary effect of the drugs in reducing the cell cycle response of the R1.40 neurons is to inhibit inflammatory processes and not to suppress or change APP processing.

In summary, neuronal CCEs are among the earliest manifestations of $\mathrm{AD}$ pathogenesis, and we argue that their presence represents a valuable biomarker for risk of neurodegeneration. The induction of these events was blocked by the chronic administration of 2 commonly used NSAIDs, ibuprofen and naproxen, when administered to animal models of the disease prior to the initial induction of neuronal CCEs. Once neuronal CCEs were initiated, however, NSAIDs were incapable of reducing the levels of cell cycle proteins within the affected cells. Our present findings suggest that successful human therapies for $\mathrm{AD}$ are best begun at the earliest possible times.

\section{Methods}

Mice. The R1.40 transgene is a full genomic copy of human APP (a 650-kb insert from a yeast artificial chromosome clone) carrying the Swedish (K670M/N671L) mutation associated with early-onset familial AD. Creation of the R1.40 transgenic mouse strain and subsequent backcrossing to inbred strains has been described previously (27-29). Age- and gender-matched nontransgenic C57BL/6J animals served as controls in all analyses. Homozygous R1.40 animals, maintained on the C57BL/6J genetic background, were crossed to Bace1 $1^{-/}$animals (provided by R. Yan, Cleveland Clinic; ref. 59), also maintained on the C57BL/6J genetic background, to generate F1 R1.40;Bace $1^{+/-}$animals. F1 animals were intercrossed to generate animals homozygous for the R1.40 transgene and homozygous for the Bace1 knockout allele as well as $\mathrm{Bace}^{-/-}$animals lacking the R1.40 transgene. Homozygous R1.40 animals, maintained on the C57BL/6J genetic background, were also crossed to $C x 3 \mathrm{cr} 1 \mathrm{gfp} / \mathrm{gf}$ animals (provided by R.M. Ransohoff, Cleveland Clinic; ref. 60), on the C57BL/6J genetic background, to generate F1 $\mathrm{R} 1.40 ; \mathrm{C} x 3 \mathrm{cr} 1^{+/ g f}$ animals. F1 animals were intercrossed to generate animals homozygous for the R1.40 transgene and heterozygous for the $\mathrm{C} x 3 \mathrm{cr} 1$ knockout allele as well as $\mathrm{C} \times 3 \mathrm{cr} 1^{+/ g f p}$ animals lacking the R1.40 transgene. Animals were housed at the Cleveland Clinic Biological Resources Unit, a facility fully 
accredited by the Association of Assessment and Accreditation of Laboratory Animal Care. All procedures were approved by the Institutional Animal Care and Use Committee of the Cleveland Clinic.

NSAID treatments. Ibuprofen and naproxen were obtained from SigmaAldrich. These compounds were formulated into standard, color-coded animal chow by Research Diets at a final concentration of 375 ppm ibuprofen or naproxen. Male and female R1.40 mice at 3 and 6 months of age and agematched nontransgenic controls were fed drug-supplemented or control chow ad libitum for either 3 or 6 months. During each trial, animals were weighed on a weekly basis. Mice were sacrificed following the experimental period and processed for either histological or biochemical analyses.

LPS administration. Male and female R1.40 mice and age-matched nontransgenic controls ( 2 months old) were injected with LPS $(20 \mu \mathrm{g} / \mathrm{animal}$, L2880; Sigma-Aldrich) or PBS via intraperitoneal injection. Animals were subject to LPS or PBS once a day for 4 consecutive days and sacrificed 4 hours after the final injection (35).

Antibodies. Antibodies used for these studies include rabbit polyclonal cyclin A antibody (ab 7956; Abcam), specific for the C-terminal domain of cyclin A2; mouse monoclonal cyclin D antibody (ab 31450; Abcam); mouse monoclonal NeuN antibody (Chemicon), used as a neuronal-specific marker; and CT-15, specific for the C-terminus of APP (diluted 1:5,000, provided by E.H. Koo, UCSD, San Diego, California, USA). Secondary antibodies were conjugated to various fluorescent Alexa Fluor dyes (Invitrogen).

Histology and immunohistochemistry. Animals were prepared for histology analysis as described previously (23). Animals were deeply anesthetized with Avertin, a mixture of 2,2,2-tribromoethanol (Sigma-Aldrich) and 2-methyl2-butanol (Sigma-Aldrich) dissolved in water at a final ratio of 1:2:80 (0.02 $\mathrm{cc} / \mathrm{mg}$ body weight); they were perfused transcardially with $0.1 \mathrm{M}$ sodium phosphate buffer, $\mathrm{pH} 7.4$, followed by $4 \%$ paraformaldehyde in $0.1 \mathrm{M}$ sodium phosphate buffer. The brain was dissected, immediately removed from the cranium, and transferred to fresh $4 \%$ paraformaldehyde at $4{ }^{\circ} \mathrm{C}$ overnight. To perform double fluorescent immunohistochemistry, sections were first rinsed in PBS containing 0.1\% Triton X-100 (PBST). Sections were subsequently incubated for 1 hour at room temperature in $10 \%$ goat serum in PBS to block nonspecific binding. All primary antibodies were diluted in PBST and applied overnight at $4^{\circ} \mathrm{C}$. After rinsing in PBS, the slides were incubated for 2 hours with a secondary antibody, which was conjugated with various fluorescent Alexa Fluor dyes (diluted 1:1,000; Invitrogen). The sections were then rinsed in PBS followed by incubation in the $10 \%$ goat serum blocking solution for 1 hour and incubation with the second primary antibody (raised in a different species than the first primary antibody) overnight at $4^{\circ} \mathrm{C}$. Sections were subsequently rinsed in PBS, and the second secondary antibody, conjugated with a difference fluorescent dye, was applied to sections for 2 hours at room temperature. After rinsing, all sections were mounted in DAPI Hardest Reagent (Vector Laboratories) under a glass coverslip. Antibody concentrations used for immunohistochemistry were as follows: rabbit anti-cyclin A2 (diluted 1:200; Abcam), mouse anti-cyclin D (diluted 1:200; Abcam), mouse anti-NeuN (diluted 1:500; Chemicon), and fluorescently tagged secondary antibodies (diluted 1:1,000; Invitrogen). FISH was performed as described previously (61) using a mouse-specific DNA probe (480C6, from the RPCI-22 BAC library) containing $150 \mathrm{~kb}$ of genomic sequence from the region that encodes the endogenous $\operatorname{Sim} 2$ gene located on mouse chromosome 16. Confocal images were acquired with a Zeiss LSM 510 META laser scanning confocal microscope (Carl Zeiss MicroImaging) using an argon laser (excitation, $488 \mathrm{~nm}$ ), a diode laser (excitation, $405 \mathrm{~nm}$ ), and a 100× Plan Apo, NA 1.4, oil immersion objective. The image represents a maximum intensity projection of a $z$ stack consisting of 14 images, each with an optical slice of $0.8 \mu \mathrm{m}$.

Image analysis. Morphometric analysis of the activation state of microglia was performed on Iba1-stained images acquired at a $\times 10$ objective using
Image-Pro Plus software (Media Cybernetics). Images were captured with a pixel depth of 8 bits, RGB, and TIFF format. The sections from different animals corresponding to the same level were imaged with identical exposure settings. At least 3 images taken at random planes covering the frontal cortex from each animal and from 3 animals per group were used for the analysis. Several earlier reports have used FF as a means to classify microglia or macrophages based on their appearance and morphological characteristics (32-34). FF measures contour irregularity of a cell by the formula $\left(4 \pi \times \mathrm{A}_{c}\right) / \mathrm{P}_{c}{ }^{2}$, where $\mathrm{A}_{c}$ and $\mathrm{P}_{c}$ represent the cell's area and perimeter, respectively. We selected $1-100$ as a filter range. FF value is higher in bushy cells, characterized by large bodies and numerous rather sparsely ramified processes, whereas cells with small, round bodies and abundant systems of regularly branched processes classified as ramified return lower FF values (32-34). In total, 583 (nontransgenic), 352 (R1.40), 188 (R1.40;Bace1-/), 555 (ibuprofen), and 686 (naproxen) Iba1-immunoreactive microglial cells per experimental group were analyzed.

As another morphometric criteria for microglial activation, the mass-radius fractal dimension was also calculated (32-34). In contrast to FF analysis, in fractal dimension analysis, cells with more ramified processes exhibit higher fractal values than bushy cells with fewer ramified processes.

Neuronal cell counts. For each treatment group, we examined 5 animals at each age. A total of 5 evenly spaced sections containing the frontal cortex were double stained for the neuronal marker NeuN and for cyclin A or cyclin $\mathrm{D}$. The area located between $2.5 \mathrm{~mm}$ and $3.4 \mathrm{~mm}$ anterior to the bregma was identified in each section analyzed. We scored NeuN-positive cells within cortical layers II/III or V/VI for the presence or absence of the cell cycle marker. Only cells with a discernible portion of their nucleus in the section were scored. For each of the 5 sections, the percentage of NeuN-positive cells exhibiting immunoreactivity for the cell cycle marker was tabulated, and the percentages for the 5 sections analyzed in each animal were averaged. For each age and treatment group, the percentages were then averaged over all 5 animals. Adjacent sections that had undergone processing for FISH were tabulated in a similar fashion, in which neurons were scored for the presence or absence of 3 or 4 spots of hybridization. All counts were performed in a blinded fashion, and data were analyzed with the Student's $t$ test, 2-tailed (GraphPad Prism, GraphPad Software).

Western blot of tissue homogenates. Mice were killed by cervical dislocation, and their brains were removed, divided sagittally (after removing cerebellum), and snap frozen. Tissues were subsequently homogenized in 10 volumes of Tris-buffered saline (50 mM Tris, $\mathrm{pH} 7.4 ; 150 \mathrm{mM} \mathrm{NaCl}$; $1 \mathrm{mM}$ EDTA; and $0.1 \%$ Triton-X) with protease inhibitor cocktail (SigmaAldrich). Total brain homogenates were subsequently sonicated to shear DNA and centrifuged at $14,000 \mathrm{~g}$ for 30 minutes at $4{ }^{\circ} \mathrm{C}$ to remove nuclei and cell debris. Total protein concentrations were determined using the Pierce bicinchoninic acid (BCA) Protein Assay Kit (Pierce). Brain protein (30 $\mu \mathrm{g}$ ) was run on a Novex NuPage, $4 \%-12 \%$ Bis-Tris gel (Invitrogen), and then transferred to a PVDF membrane. The Western blots were subsequently incubated with antibody CT-15 against the C terminus of APP (62).

A $\beta$ ELISA. Mouse brain hemispheres were extracted with $70 \%$ formic acid, homogenized at $100,000 \mathrm{~g}$, and neutralized (to $\mathrm{pH} 7.5$ ) with $10 \times 2 \mathrm{M}$ Tris ( $\mathrm{pH}$. 11.0). Brain extracts were frozen once and then tested for $A \beta_{1-40}$ and $A \beta_{1-42}$ levels. $A \beta_{1-40}$ and $A \beta_{1-42}$ were measured in brain extracts using specific sandwich ELISAs developed for mouse brain tissue containing the human APP transcript. $A \beta$ peptides were captured using either the $A \beta_{1-40^{-}}$ selective monoclonal antibody $\mathrm{B} 113$ or the $\mathrm{A} \beta_{1-42}$-selective monoclonal antibody A387. Bound peptides were detected using alkaline phosphatase mAb B436 (raised against $A \beta_{1-12}$ ) combined with a CSPD-Sapphire II Luminescence substrate (Applied Biosystems) in order to quantify alkaline phosphatase activity. Relative luminescent units were measured using a standard 96-well luminometer. Each sample was assayed in triplicate 
at appropriate dilutions so that relative luminescent units were in the standard curve range included on each plate. Standard curves were constructed with synthetic $A \beta_{1-40}$ or $A \beta_{1-42}$ peptides (Bachem) spiked into $\mathrm{C} 57 \mathrm{BL} / 6 \mathrm{~J}$ brain extract and diluted in a manner similar to that for the samples using medium.

Statistics. Data are presented as mean \pm SEM unless otherwise specified. Differences between groups were determined by unpaired Student's $t$ test, 2-tailed. A $P$ value less than 0.05 was considered significant.

\section{Acknowledgments}

This work was supported by NIH grants AG023012 (to B.T. Lamb) and AGO24494 (to K. Herrup and B.T. Lamb), the Alzheimer's Association (to B.T. Lamb), and an anonymous foundation. We thank R.M. Ransohoff for C $x 3 \mathrm{cr} 1 g \mathrm{fp} / \mathrm{gp}$ mice and R. Yan for Bace1 ${ }^{-/-}$ mice. We thank Maryanne Pendergast and the Case Western
Reserve University Neurosciences Imaging Center for assistance with the confocal microscopy.

Received for publication May 1, 2009, and accepted in revised form September 16, 2009.

Address correspondence to: Karl Herrup, Rutgers, The State University of New Jersey, Department of Cell Biology and Neuroscience, Nelson Biological Laboratories, Busch Campus, 604 Allison Road, Piscataway, New Jersey 08854-8082, USA. Phone: (732) $445-$ 1794; Fax: (732) 445-3306; E-mail: herrup@biology.rutgers.edu. Or to: Bruce T. Lamb, Lerner Research Institute, Cleveland Clinic, Department of Neurosciences NC3-164, 9500 Euclid Avenue, NC30, Cleveland, Ohio 44195-0001, USA. Phone: (216) 444-3592; Fax: (216) 444-7927; E-mail: lambb@ccf.org.
1. Alzheimer's Association. 2009. 2009 Alzheimer's disease facts and figures. Alzheimers Dement. 5:234-270.

2. Akiyama, H., et al. 2000. Inflammation and Alzheimer's disease. Neurobiol. Aging. 21:383-421.

3. Wyss-Coray, T. 2006. Inflammation in Alzheimer disease: driving force, bystander or beneficial response? Nat. Med. 12:1005-1015.

4. Bolmont, T., et al. 2008. Dynamics of the microglial/amyloid interaction indicate a role in plaque maintenance. J. Neurosci. 28:4283-4292.

5. Meyer-Luehmann, M., et al. 2008. Rapid appearance and local toxicity of amyloid-beta plaques in a mouse model of Alzheimer's disease. Nature. 451:720-724.

6. McGeer, P.L., Schulzer, M., and McGeer, E.G. 1996. Arthritis and anti-inflammatory agents as possible protective factors for Alzheimer's disease: a review of 17 epidemiologic studies. Neurology. 47:425-432.

7. Stewart, W.F., Kawas, C., Corrada, M., and Metter, E.J. 1997. Risk of Alzheimer's disease and duration of NSAID use. Neurology. 48:626-632.

8. Vlad, S.C., Miller, D.R., Kowall, N.W., and Felson, D.T. 2008. Protective effects of NSAIDs on the development of Alzheimer disease. Neurology. 70:1672-1677.

9. Martin, B.K., et al. 2008. Cognitive function over time in the Alzheimer's Disease Anti-inflammatory Prevention Trial (ADAPT): results of a randomized, controlled trial of naproxen and celecoxib. Arch. Neurol. 65:896-905.

10. Lim, G.P., et al. 2001. Ibuprofen effects on Alzheimer pathology and open field activity in APPsw transgenic mice. Neurobiol. Aging. 22:983-991.

11. Lim, G.P., et al. 2000. Ibuprofen suppresses plaque pathology and inflammation in a mouse model for Alzheimer's disease. J. Neurosci. 20:5709-5714.

12. Kukar, T., et al. 2007. Chronic administration of R-flurbiprofen attenuates learning impairments in transgenic amyloid precursor protein mice. $B M C$ Neurosci. 8:54.

13. Sung, S., et al. 2004. Modulation of nuclear factorkappa B activity by indomethacin influences Abeta levels but not Abeta precursor protein metabolism in a model of Alzheimer's disease. Am. J. Pathol. 165:2197-2206.

14. Eriksen, J., et al. 2003. NSAIDs and enantiomers of flurbiprofen target gamma-secretase and lower Abeta 42 in vivo. J. Clin. Invest. 112:440-449.

15. Weggen, S., et al. 2001. A subset of NSAIDs lower amyloidogenic Abeta42 independently of cyclooxygenase activity. Nature. 414:212-216.

16. Kukar, T.L., et al. 2008. Substrate-targeting gammasecretase modulators. Nature. 453:925-929.

17. Sastre, M., et al. 2006. Nonsteroidal anti-inflammatory drugs repress beta-secretase gene promoter activity by the activation of PPARgamma. Proc. Natl. Acad. Sci. U. S. A. 103:443-448.

18. Arendt, T., Rodel, L., Gartner, U., and Holzer, M.
1996. Expression of the cyclin-dependent kinase inhibitor p16 in Alzheimer's disease. Neuroreport. 7:3047-3049.

19. McShea, A., Harris, P.L., Webster, K.R., Wahl, A.F., and Smith, M.A. 1997. Abnormal expression of the cell cycle regulators P16 and CDK4 in Alzheimer's disease. Am. J. Pathol. 150:1933-1939.

20. Vincent, I., Rosado, M., and Davies, P. 1996. Mitotic mechanisms in Alzheimer's disease? J. Cell Biol. 132:413-425.

21. Yang, Y., Geldmacher, D.S., and Herrup, K. 2001. DNA replication precedes neuronal cell death in Alzheimer's disease. J. Neurosci. 21:2661-2668.

22. Petersen, R.C. 2000. Mild cognitive impairment: transition between aging and Alzheimer's disease. Neurologia. 15:93-101.

23. Yang, Y., Varvel, N.H., Lamb, B.T., and Herrup, K. 2006. Ectopic cell cycle events link human Alzheimer's disease and amyloid precursor protein transgenic mouse models. J. Neurosci. 26:775-784.

24. Kim, D., et al. 2008. Deregulation of HDAC1 by p25/Cdk5 in neurotoxicity. Neuron. 60:803-817.

25. Kallhoff-Munoz, V., Hu, L., Chen, X., Pautler, R.G., and Zheng, H. 2008. Genetic dissection of gammasecretase-dependent and -independent functions of presenilin in regulating neuronal cell cycle and cell death. J. Neurosci. 28:11421-11431.

26. Andorfer, C., et al. 2005. Cell-cycle reentry and cell death in transgenic mice expressing nonmutant human tau isoforms. J. Neurosci. 25:5446-5454

27. Lamb, B.T., et al. 1997. Altered metabolism of familial Alzheimer's disease-linked amyloid precursor protein variants in yeast artificial chromosome transgenic mice. Hum. Mol. Genet. 6:1535-1541.

28. Lamb, B.T., et al. 1993. Introduction and expression of the 400 kilobase amyloid precursor protein gene in transgenic mice. Nat. Genet. 5:22-30.

29. Lehman, E.J., et al. 2003. Genetic Background Regulates beta-Amyloid Precursor Protein Processing and beta-Amyloid Deposition in the Mouse. Hum. Mol. Genet. 12:2949-2956.

30. Varvel, N.H., et al. 2008. Abeta oligomers induce neuronal cell cycle events in Alzheimer's disease. J. Neurosci. 28:10786-10793.

31. Kulnane, L.S., and Lamb, B.T. 2001. Neuropathological characterization of mutant amyloid precursor protein yeast artificial chromosome transgenic mice. Neurobiol. Dis. 8:982-992.

32. Soltys, Z., et al. 2005. Quantitative morphological study of microglial cells in the ischemic rat brain using principal component analysis. J. Neurosci. Methods. 146:50-60.

33. Orlowski, D., Soltys, Z., and Janeczko, K. 2003. Morphological development of microglia in the postnatal rat brain. A quantitative study. Int. J. Dev. Neurosci. 21:445-450.

34. Soltys, Z., Ziaja, M., Pawlinski, R., Setkowicz, Z., and Janeczko, K. 2001. Morphology of reactive microglia in the injured cerebral cortex. Fractal analysis and complementary quantitative methods. J. Neurosci. Res. 63:90-97.

35. Cardona, A.E., et al. 2006. Control of microglial neurotoxicity by the fractalkine receptor. Nat. Neurosci. 9:917-924.

36. Mintun, M.A., et al. 2006. [11C]PIB in a nondemented population: potential antecedent marker of Alzheimer disease. Neurology. 67:446-452.

37. Rowe, C.C., et al. 2007. Imaging beta-amyloid burden in aging and dementia. Neurology. 68:1718-1725.

38. Troncoso, J.C., Martin, L.J., Dal Forno, G., and Kawas, C.H. 1996. Neuropathology in controls and demented subjects from the Baltimore Longitudinal Study of Aging. Neurobiol. Aging. 17:365-371.

39. Snowdon, D.A. 1997. Aging and Alzheimer's disease: lessons from the Nun Study. Gerontologist. 37:150-156.

40. Busser, J., Geldmacher, D.S., and Herrup, K. 1998. Ectopic cell cycle proteins predict the sites of neuronal cell death in Alzheimer's disease brain. J. Neurosci. 18:2801-2807.

41. Yang, Y., Mufson, E.J., and Herrup, K. 2003. Neuronal cell death is preceded by cell cycle events at all stages of Alzheimer's disease. J. Neurosci. 23:2557-2563.

42. Janelsins, M.C., et al. 2005. Early correlation of microglial activation with enhanced tumor necrosis factor-alpha and monocyte chemoattractant protein-1 expression specifically within the entorhinal cortex of triple transgenic Alzheimer's disease mice. J. Neuroinflammation. 2:23.

43. Tehranian, R., Hasanvan, H., Iverfeldt, K., Post, C., and Schultzberg, M. 2001. Early induction of interleukin-6 mRNA in the hippocampus and cortex of APPsw transgenic mice Tg2576. Neurosci. Lett. 301:54-58.

44. Simard, A.R., Soulet, D., Gowing, G., Julien, J.P., and Rivest, S. 2006. Bone marrow-derived microglia play a critical role in restricting senile plaque formation in Alzheimer's disease. Neuron. 49:489-502.

45. El Khoury, J., et al. 2007. Ccr2 deficiency impairs microglial accumulation and accelerates progression of Alzheimer-like disease. Nat. Med. 13:432-438.

46. Town, T., et al. 2008. Blocking TGF-beta-Smad2/3 innate immune signaling mitigates Alzheimer-like pathology. Nat. Med. 14:681-687.

47. Etminan, M., Gill, S., and Samii, A. 2003. Effect of non-steroidal anti-inflammatory drugs on risk of Alzheimer's disease: systematic review and metaanalysis of observational studies. BMJ. 327:128.

48. Kotilinek, L.A., et al. 2008. Cyclooxygenase-2 inhibition improves amyloid-beta-mediated suppression of memory and synaptic plasticity. Brain. 131:651-664

49. Yan, Q., et al. 2003. Anti-inflammatory drug therapy alters beta-amyloid processing and deposition 
in an animal model of Alzheimer's disease. J. Neurosci. 23:7504-7509.

50. Alafuzoff, I., Overmyer, M., Helisalmi, S., and Soininen, H. 2000. Lower counts of astroglia and activated microglia in patients with Alzheimer's disease with regular use of non-steroidal antiinflammatory drugs. J. Alzheimers Dis. 2:37-46.

51. Mackenzie, I.R., and Munoz, D.G. 1998. Nonsteroidal anti-inflammatory drug use and Alzheimertype pathology in aging. Neurology. 50:986-990.

52. Heneka, M.T., et al. 2005. Acute treatment with the PPARgamma agonist pioglitazone and ibuprofen reduces glial inflammation and Abeta1-42 levels in APPV717I transgenic mice. Brain. 128:1442-1453.

53. Jantzen, P.T., et al. 2002. Microglial activation and beta -amyloid deposit reduction caused by a nitric oxide-releasing nonsteroidal anti-inflammatory drug in amyloid precursor protein plus presenilin-1 transgenic mice. J. Neurosci. 22:2246-2254.

54. Aisen, P.S., et al. 2003. Effects of rofecoxib or naproxen vs placebo on Alzheimer disease progression: a randomized controlled trial. JAMA. 289:2819-2826.

55. Green, R.D., Schneider, L.S., Hendrix, S.B., Zavitz, K.H., and Swabb, E. 2008. Safety and efficacy of tarenflurbil in subjects with mild Alzheimer's disease: results from an 18-month multi-center phase 3 trial. Alzheimers Dement. 4(Suppl.):T165.

56. Kukar, T., et al. 2005. Diverse compounds mimic Alzheimer disease-causing mutations by augmenting Abeta42 production. Nat. Med. 11:545-550.

57. Lanz, T.A., Fici, G.J., and Merchant, K.M. 2005. Lack of specific amyloid-beta(1-42) suppression by nonsteroidal anti-inflammatory drugs in young, plaque-free $\operatorname{Tg} 2576$ mice and in guinea pig neuronal cultures. J. Pharmacol. Exp. Ther. 312:399-406.

58. Lehmann, J.M., Lenhard, J.M., Oliver, B.B., Ringold,
G.M., and Kliewer, S.A. 1997. Peroxisome proliferator-activated receptors alpha and gamma are activated by indomethacin and other non-steroidal antiinflammatory drugs. J. Biol. Chem. 272:3406-3410.

59. Cai, H., et al. 2001. BACE1 is the major beta-secretase for generation of Abeta peptides by neurons. Nat. Neurosci. 4:233-234.

60. Geissmann, F., Jung, S., and Littman, D.R. 2003. Blood monocytes consist of two principal subsets with distinct migratory properties. Immunity. 19:71-82.

61. Kulnane, L.S., Lehman, E.J., Hock, B.J., Tsuchiya, K.D., and Lamb, B.T. 2002. Rapid and efficient detection of transgene homozygosity by FISH of mouse fibroblasts. Mamm. Genome. 13:223-226.

62. Martin, L.J., et al. 1991. Amyloid precursor protein in aged nonhuman primates. Proc. Natl. Acad. Sci. U. S. A. 88:1461-1465. 